\title{
Improved Modified Ratio Estimation of Population Mean Using Information on Size of the Sample
}

\author{
Sikiru A Suleiman ${ }^{1 *}$ and Amos A Adewara ${ }^{2}$ \\ ${ }^{1}$ Department of Statistics, Kwara State Polytechnic, Ilorin, Nigeria \\ ${ }^{2}$ Department of Statistics, University of Ilorin, Ilorin, Nigeria \\ *Corresponding author email: sikirusuleiman097@gmail.com
}

Received 25 Jul 2021, Revised 2 Dec 2021, Accepted 6 Dec 2021, Published Dec 2021

DOI: https://dx.doi.org/10.4314/tjs.v47i5.22

\begin{abstract}
In sample surveys, auxiliary information is used for estimation to improve the efficiency of estimators. Increased precision can be obtained when the variable under study is highly correlated with auxiliary information. In this study, the sample size has been used as information for improved estimation of population mean of the main variable under study. A new modified generalized ratio type estimator of population mean has been proposed and the efficiency was examined using Murthy (1967) and Mukhopadhyay (2009) dataset. The large sample properties, the bias and the mean squared error of the newly proposed modified ratio estimator were obtained up to first order of approximation. The optimum value of the characterizing scalar which minimizes the mean squared error was obtained and the minimum value of the mean squared error of the proposed modified ratio estimator for this optimum value was also obtained. A theoretical comparison of the proposed modified ratio estimators was made with the other existing related estimators of population mean using auxiliary information. The conditions under which the proposed modified ratio estimators perform better than the other existing estimators of population mean are given. A numerical study was also carried out to see the performances of the proposed modified ratio estimators and some existing related ratio estimators of population mean and verify the conditions under which the proposed modified ratio estimators are better than some other existing related ratio estimators considered. It was shown that the proposed modified ratio estimators perform better than some existing related ratio estimators as they are having lower mean squared errors.
\end{abstract}

Keywords: Ratio Estimator, Sample size, Bias, Mean Squared Error, Efficiency.

\section{Introduction}

In sampling theory, estimation of the population parameters is necessary when the size of the population is very large (Gupta and Yadav 2018) and we wish to get the results in very shortest time and with minimum costs, fewer labor, etc. In order to estimate any parameter, the best estimator is the corresponding statistic. Thus, the sample mean is the most suitable estimator for estimating population mean, but it has a reasonably large sampling variance (Gupta and Yadav 2017). Our purpose is to search for the estimator with higher efficiency that has minimum variance or mean squared error. This aim is achieved through the use of auxiliary information provided by the auxiliary variables or auxiliary attributes. It is a well-established phenomenon that supplementary information provided by auxiliary variables often improves the accuracy of estimators of unknown population parameters. Ratio, product, and regression-type estimators are three such methods. Auxiliary information is obtained from auxiliary variable which is highly 
positively or negatively correlated with the main variable under study (Gupta and Yadav 2017).

Let the finite population under consideration consist of $N$ distinct and identifiable units, and let ( $x i, y i), i=1,2, \ldots, n$ be a two variable sample of size $n$ taken from bivariate variables ( $\mathrm{X}, \mathrm{Y}$ ) through simple random sampling without sampling scheme. Let $\bar{X}$ and $\bar{Y}$ be the population means of the auxiliary and the study variables, respectively, and let $\bar{x}$ and $\bar{y}$ be the respective sample means and both are unbiased estimators of $\bar{X}$ and $\bar{Y}$, respectively. Let the correlation coefficient between the variables $X$ and $Y$ be denoted by $\rho$.

In this study, we have confined our work to positively correlated populations only and proposed seven ratio type estimators for improved estimation of the population mean with higher efficiencies. In addition, its large sample properties have been studied up to the first order of approximation. In sampling literature, many estimators have been proposed when a single auxiliary variable is involved, and they are found to be more efficient than the sample mean, the ratio and product estimators under some realistic conditions, as well as efficient as the regression estimator in the optimum case but the problem of the best estimator in terms of both efficiency and biasedness has not been fully exhausted. This work was another attempt in solving this problem. The aim of this research work was to improve the efficiency of some modified existing ratio type estimators of population mean using suitably chosen scalar such that the mean squared error of the proposed estimator is minimum.

\section{Literature Review}

Let $U$ denote a finite population consisting of $\mathrm{N}$ units $\left\{U_{l}, U_{2}, \ldots, U_{N}\right\}$. Also let $\mathrm{Y}$ be study variable taking values $\left\{Y_{l}, Y_{2}, \ldots, Y_{N}\right\}$ and $\mathrm{X}$ be auxiliary variable taking values $\left\{X_{1}\right.$, $\left.X_{2}, \ldots, X_{N}\right\}$ on $\mathrm{i}^{\text {th }}$ unit $U_{i}$ of the population $U$.

$\bar{Y}=\frac{1}{N} \sum_{i=1}^{N} Y_{i}$-population mean of the study variable $\mathrm{Y}$.
$\bar{X}=\frac{1}{N} \sum_{i=1}^{N} X_{i} \quad$-population mean of the auxiliary variable $\mathrm{X}$.

$\bar{y}=\frac{1}{n} \sum_{i=1}^{n} y_{i}$-sample mean of the study variable Y.

$\bar{x}=\frac{1}{n} \sum_{i=1}^{n} x_{i}$-sample mean of the auxiliary variable $\mathrm{X}$.

$S_{y}^{2}=\frac{1}{N-1} \sum_{i=1}^{N}\left(Y_{i}-\bar{Y}\right)^{2}$-finite population variance of study variable $\mathrm{Y}$.

$S_{x}^{2}=\frac{1}{N-1} \sum_{i=1}^{N}\left(X_{i}-\bar{X}\right)^{2}$-finite $\quad$ population variance of auxiliary variable $\mathrm{X}$.

$S_{y x}=\frac{1}{N-1} \sum_{i=1}^{N}\left(Y_{i}-\bar{Y}\right)\left(X_{i}-\bar{X}\right)$-finite

population covariance of $\mathrm{X}$ and $\mathrm{Y}$.

$\rho_{y x}=\frac{S_{y x}}{S_{x} S_{y}}$-Pearson's moment correlation coefficient of $\mathrm{X}$ and $\mathrm{Y}$.

$C_{y}=\frac{S_{y}}{\bar{Y}}$-coefficient of variation of $\mathrm{Y}$.

$C_{x}=\frac{S_{x}}{\bar{X}}$-coefficient of variation of X.

$M_{d}$-Median of the auxiliary variable X.

$\beta_{1}=\frac{N \sum_{i=1}^{N}\left(X_{i}-\bar{X}\right)^{3}}{(N-1)(N-2) S_{x}^{3}}$-coefficient of skewness of auxiliary variable $\mathrm{X}$.

$\beta_{2}=\frac{N(N+1) \sum_{i=1}^{N}\left(X_{i}-\bar{X}\right)^{4}}{(N-1)(N-2)(N-3) S_{x}^{4}}-\frac{3(N-1)^{2}}{(N-2)(N-3)}-$

coefficient of kurtosis of auxiliary variable $\mathrm{X}$.

\section{Review of existing estimators}

As mentioned earlier, the most suitable estimator for estimating population mean $\bar{Y}$ is the sample mean $\bar{y}$ given by,

$$
\bar{y}=\frac{1}{n} \sum_{i=1}^{n} y_{i}
$$

It is unbiased for population mean and its variance up to the first order of approximation is given by, 
$V(\bar{y})=\frac{1-f}{n} S_{y}^{2}=\frac{1-f}{n} \bar{Y}^{2} C_{y}^{2}=\gamma \bar{Y}^{2} C_{y}^{2} \quad C_{y}=\frac{S_{y}}{\bar{Y}^{2}}, S_{y}^{2}=\frac{1}{N-1} \sum_{i=1}^{N}\left(Y_{i}-\bar{Y}\right)^{2}, \gamma=\frac{1-f}{n}, f=\frac{n}{N}$

(2)

where,

Table 1: Biases and mean squared errors (MSE) of some existing modified ratio estimators

\begin{tabular}{|c|c|c|c|c|}
\hline S/No & Estimator & Constant & Bias & MSE \\
\hline 1. & $\begin{array}{l}\hat{\bar{Y}}_{1}=\bar{y}\left(\frac{\bar{X}+C_{x}}{\bar{x}+C_{x}}\right) \\
\text { Sisodia and Dwivedi } \\
\text { (1981) }\end{array}$ & $\delta_{1}=\left(\frac{\bar{X}}{\bar{X}+C_{x}}\right)$ & $\gamma \bar{Y}\left(\delta_{1}^{2} C_{x}^{2}-2 \delta_{1} \rho C_{x} C_{y}\right)$ & $\gamma \bar{Y}^{2}\left(\begin{array}{l}C_{y}^{2}+\delta_{1}^{2} C_{x}^{2} \\
-2 \delta_{1} \rho C_{x} C_{y}\end{array}\right)$ \\
\hline 2. & $\begin{array}{l}\hat{\bar{Y}}_{2}=\bar{y}\left(\frac{\bar{X} C_{x}+\beta_{2}}{\bar{x} C_{x}+\beta_{2}}\right) \\
\text { Upadhyaya and Singh } \\
\text { (1999) }\end{array}$ & $\delta_{2}=\left(\frac{\bar{X} C_{x}}{\bar{X} C_{x}+\beta_{2}}\right)$ & $\gamma \bar{Y}\left(\delta_{2}^{2} C_{x}^{2}-2 \delta_{2} \rho C_{x} C_{y}\right)$ & $\gamma \bar{Y}^{2}\left(\begin{array}{l}C_{y}^{2}+\delta_{2}^{2} C_{x}^{2} \\
-2 \delta_{2} \rho C_{x} C_{y}\end{array}\right)$ \\
\hline 3. & $\begin{array}{l}\hat{\bar{Y}}_{3}=\bar{y}\left(\frac{\bar{X}+\rho}{\bar{x}+\rho}\right) \\
\text { Singh and Tailor } \\
(2003)\end{array}$ & $\delta_{3}=\left(\frac{\bar{X}}{\bar{X}+\rho}\right)$ & $\gamma \bar{Y}\left(\delta_{3}^{2} C_{x}^{2}-2 \delta_{3} \rho C_{x} C_{y}\right)$ & $\gamma \bar{Y}^{2}\left(\begin{array}{l}C_{y}^{2}+\delta_{3}^{2} C_{x}^{2} \\
-2 \delta_{3} \rho C_{x} C_{y}\end{array}\right)$ \\
\hline 4. & $\begin{array}{l}\hat{\bar{Y}}_{4}=\bar{y}\left(\frac{\bar{X}+\beta_{2}}{\bar{x}+\beta_{2}}\right) \\
\text { Singh et al. (2004) }\end{array}$ & $\delta_{4}=\left(\frac{\bar{X}}{\bar{X}+\beta_{2}}\right)$ & $\gamma \bar{Y}\left(\delta_{4}^{2} C_{x}^{2}-2 \delta_{4} \rho C_{x} C_{y}\right)$ & $\gamma \bar{Y}^{2}\left(\begin{array}{l}C_{y}^{2}+\delta_{4}^{2} C_{x}^{2} \\
-2 \delta_{4} \rho C_{x} C_{y}\end{array}\right)$ \\
\hline 5. & $\begin{array}{l}\hat{\bar{Y}}_{5}=\bar{y}\left(\frac{\bar{X}+\beta_{1}}{\bar{x}+\beta_{1}}\right) \\
\text { Yan and Tian (2010) }\end{array}$ & $\delta_{5}=\left(\frac{\bar{X}}{\bar{X}+\beta_{1}}\right)$ & $\gamma \bar{Y}\left(\delta_{5}^{2} C_{x}^{2}-2 \delta_{5} \rho C_{x} C_{y}\right)$ & $\gamma \bar{Y}^{2}\left(\begin{array}{l}C_{y}^{2}+\delta_{5}^{2} C_{x}^{2} \\
-2 \delta_{5} \rho C_{x} C_{y}\end{array}\right)$ \\
\hline 6. & $\begin{array}{l}\hat{\bar{Y}}_{6}=\bar{y}\left(\frac{\bar{X}+M_{d}}{\bar{x}+M_{d}}\right) \\
\text { Subramani and } \\
\text { Kumarpandiyan } \\
\text { (2013) }\end{array}$ & $\delta_{6}=\left(\frac{\bar{X}}{\bar{X}+M_{d}}\right)$ & $\gamma \bar{Y}\left(\delta_{6}^{2} C_{x}^{2}-2 \delta_{6} \rho C_{x} C_{y}\right)$ & $\gamma \bar{Y}^{2}\left(\begin{array}{l}C_{y}^{2}+\delta_{6}^{2} C_{x}^{2} \\
-2 \delta_{6} \rho C_{x} C_{y}\end{array}\right)$ \\
\hline 7 & $\begin{array}{l}\hat{\bar{Y}}_{7}=\bar{y}\left(\frac{\bar{X}+n}{\bar{x}+n}\right) \\
\text { Jerajuddin and Kishun } \\
(2016)\end{array}$ & $\delta_{7}=\left(\frac{\bar{X}}{\bar{X}+n}\right)$ & $\gamma \bar{Y}\left(\delta_{7}^{2} C_{x}^{2}-2 \delta_{7} \rho C_{x} C_{y}\right)$ & $\gamma \bar{Y}^{2}\left(\begin{array}{l}C_{y}^{2}+\delta_{7}^{2} C_{x}^{2} \\
-2 \delta_{7} \rho C_{x} C_{y}\end{array}\right)$ \\
\hline
\end{tabular}

Cochran (1940) used the positively correlated auxiliary variable with the study variable and proposed the following usual ratio estimator of population mean as,

$$
\hat{\bar{Y}}_{r}=\bar{y} \frac{\bar{X}}{\bar{x}}
$$

The above estimator is a biased estimator of population mean and its bias and mean squared error, up to the first order of approximation, respectively are,
$B\left(\hat{\bar{Y}}_{r}\right)=\frac{1-f}{n} \bar{Y}\left[C_{y}^{2}-\rho_{y x} C_{y} C_{x}\right]$

$\operatorname{MSE}\left(\hat{\bar{Y}}_{r}\right)=\frac{1-f}{n} \bar{Y}^{2}\left[C_{y}^{2}+C_{x}^{2}-2 \rho_{y x} C_{y} C_{x}\right]$

Where,

$$
\begin{gathered}
C_{x}=\frac{S_{x}}{\bar{X}}, S_{x}^{2}=\frac{1}{N-1} \sum_{i=1}^{N}\left(X_{i}-\bar{X}\right)^{2}, \rho_{y x}=\frac{\operatorname{Cov}(x, y)}{S_{x} S_{y}}, \\
\operatorname{Cov}(x, y)=\frac{1}{N-1} \sum_{i=1}^{N}\left(Y_{i}-\bar{Y}\right)\left(X_{i}-\bar{X}\right),
\end{gathered}
$$

In literature, various modified estimators of population mean of the study variable using auxiliary variables have been given by 
various authors. For detailed study of the modified ratio type estimators, latest references can be made of Kadilar and Cingi (2004, 2006(a, b), 2009), Singh (2003), Singh and Tailor (2003, 2005), Singh and Chaudhary (1986), Gupta and Misra (2006), Gupta and Yadav (2017 and 2018), Koyuncu and Kadilar (2009), Misra and Gupta (2008), Subramani (2013a), Subramani and Kumarapandiyan (2012(a,b,c), 2013, Subramani (2013b)), Tailor and Sharma (2009), Yan and Tian (2010), Yadav and Pandey (2011), Yadav and Adewara (2013), Yadav et al. (2014, 2015), Yadav et al. (2016(a, b, c, d)), Abid et al. (2016), Misra et al. (2012), Jerajuddin and Kishun (2016), Cochran (1977) and Tailor et al. (2011).

Thus, biases and mean squared errors of the above estimators may be written as,

$$
\begin{aligned}
& B(\hat{\bar{Y}})=\gamma \bar{Y}\left(\delta_{i}^{2} C_{x}^{2}-2 \delta_{i} \rho C_{x} C_{y}\right), \\
& \operatorname{MSE}(\hat{\bar{Y}})=\gamma \bar{Y}^{2}\left(C_{y}^{2}+\delta_{i}^{2} C_{x}^{2}-2 \delta_{i} \rho C_{x} C_{y}\right), i=1,2, \ldots, 7 .
\end{aligned}
$$

\section{Materials and Methods}

\section{The proposed estimators}

Motivated by Sisodia and Dwivedi (1981), Upadhyaya and Singh (1999), Singh and Tailor (2003), Singh et al. (2004), Yan and Tian (2010), Subramani and Kumarpandiyan (2013), Jerajuddin and Kishun (2016) and Gupta and Yadav (2018) estimator of population mean, the following generalized estimators of the population mean using information on size of the sample were proposed as,

$$
\begin{aligned}
& \xi_{p 1}=\bar{y}\left[\alpha_{1}+\left(1-\alpha_{1}\right)\left(\frac{\bar{X}+C_{x} n}{\bar{x}+C_{x} n}\right)\right] \\
& \xi_{p 2}=\bar{y}\left[\alpha_{2}+\left(1-\alpha_{2}\right)\left(\frac{\bar{X} C_{x}+\beta_{2} n}{\bar{x} C_{x}+\beta_{2} n}\right)\right] \\
& \xi_{p 3}=\bar{y}\left[\alpha_{3}+\left(1-\alpha_{3}\right)\left(\frac{\bar{X}+\rho n}{\bar{x}+\rho n}\right)\right] \\
& \xi_{p 4}=\bar{y}\left[\alpha_{4}+\left(1-\alpha_{4}\right)\left(\frac{\bar{X}+\beta_{2} n}{\bar{x}+\beta_{2} n}\right)\right]
\end{aligned}
$$

$$
\begin{aligned}
& \xi_{p 5}=\bar{y}\left[\alpha_{5}+\left(1-\alpha_{5}\right)\left(\frac{\bar{X}+\beta_{1} n}{\bar{x}+\beta_{1} n}\right)\right] \\
& \xi_{p 6}=\bar{y}\left[\alpha_{6}+\left(1-\alpha_{6}\right)\left(\frac{\bar{X}+M_{d} n}{\bar{x}+M_{d} n}\right)\right] \\
& \xi_{p 7}=\bar{y}\left[\alpha_{7}+\left(1-\alpha_{7}\right)\left(\frac{\bar{X} M_{d}+n}{\bar{x} M_{d}+n}\right)\right]
\end{aligned}
$$

Where, $\alpha_{i}(i=1,2, \ldots, 7)$ is a suitably chosen constant to be defined such that the mean squared error of the proposed estimator is minimum.

To study the large sample properties of the proposed modified ratio estimators, we have used the following approximations as:

$\bar{y}=\bar{Y}\left(1+e_{0}\right) ; \quad \bar{x}=\bar{X}\left(1+e_{1}\right) \quad$ such that $E\left(e_{i}\right)=0,(i=0,1)$ and $E\left(e_{0}^{2}\right)=\frac{1-f}{n} C_{y}^{2}$

and $E\left(e_{1}^{2}\right)=\frac{1-f}{n} C_{x}^{2}, E\left(e_{0} e_{1}\right)=\frac{1-f}{n} \rho C_{x} C_{y}$

\section{Bias and MSE of $\xi_{p 1}$}

Expressing Equation (6) in terms of $e_{i}{ }^{\prime} s$, we get

$\xi_{p 1}=\bar{Y}\left(1+e_{0}\right)\left[\alpha_{1}+\left(1-\alpha_{1}\right)\left(\frac{\bar{X}+C_{x} n}{\bar{X}\left(1+e_{1}\right)+C_{x} n}\right)\right]$

$\xi_{p 1}=\bar{Y}\left(1+e_{0}\right)\left[\alpha_{1}+\left(1-\alpha_{1}\right)\left(\frac{1}{1+\frac{\bar{X}}{\bar{X}+C_{x} n} e_{1}}\right)\right]$

$\xi_{1}=\bar{Y}\left(1+e_{0}\right)\left[\alpha_{1}+\left(1-\alpha_{1}\right)\left(1+\delta_{p 1} e_{1}\right)^{-1}\right]$

Where, $\delta_{p 1}=\frac{\bar{X}}{\bar{X}+C_{x} n}$

We assume that $\left|e_{1}\right|<1$, so that $\left(1+\delta_{p 1} e_{1}\right)^{-1}$ may be expanded. Now expanding the right-hand side of Equation (13), we have,

$$
\xi_{p 1}=\bar{Y}\left(1+e_{0}\right)\left[\alpha_{1}+\left(1-\alpha_{1}\right)\left(1+\delta_{p 1} e_{1}+\delta_{p 1}^{2} e_{1}^{2}\right)\right]
$$

$$
\xi_{p 1}=\bar{Y}\left(1+e_{0}\right)\left[\alpha_{1}+1-\delta_{p 1} e_{1}+\delta_{p 1}^{2} e_{1}^{2}-\alpha_{1}+\alpha_{1} \delta_{p 1} e_{1}-\alpha_{1} \delta_{p 1}^{2} e_{1}^{2}\right\rfloor
$$




$$
\xi_{p 1}=\bar{Y}\left(1+e_{0}\right)\left\lfloor 1-\delta_{p 1} e_{1}+\delta_{p 1}^{2} e_{1}^{2}+\alpha_{1} \delta_{p 1} e_{1}-\alpha_{1} \delta_{p 1}^{2} e_{1}^{2}\right\rfloor
$$

Retaining the terms up to the first order of approximation, we have

$$
\xi_{p 1}=\bar{Y}\left[1+e_{0}-\delta_{p 1} e_{1}-\delta_{p 1} e_{0} e_{1}+\delta_{p 1}^{2} e_{1}^{2}+\alpha_{1} \delta_{p 1} e_{1}+\alpha_{1} \delta_{p 1} e_{0} e_{1}-\alpha_{1} \delta_{p 1}^{2} e_{1}^{2}\right]
$$

Subtracting $\bar{Y}$ from both sides of Equation (16), we get

$$
\xi_{p 1}-\bar{Y}=\bar{Y}\left[e_{0}-\delta_{p 1} e_{1}-\delta_{p 1} e_{0} e_{1}+\delta_{p 1}^{2} e_{1}^{2}+\alpha_{1} \delta_{p 1} e_{1}+\alpha_{1} \delta_{p 1} e_{0} e_{1}-\alpha_{1} \delta_{p 1}^{2} e_{1}^{2}\right\rfloor
$$

Taking expectations on both sides of Equation (17) and putting the values of different expectations, we get the bias of $\xi_{p 1}$ as

$$
\begin{aligned}
& E\left(\xi_{p 1}-\bar{Y}\right)=B\left(\xi_{p 1}\right)=\bar{Y} E\left[e_{0}-\delta_{p 1} e_{1}-\delta_{p 1} e_{0} e_{1}+\delta_{p 1}^{2} e_{1}^{2}+\alpha_{1} \delta_{p 1} e_{1}+\alpha_{1} \delta_{p 1} e_{0} e_{1}-\alpha_{1} \delta_{p 1}^{2} e_{1}^{2}\right] \\
& B\left(\xi_{p 1}\right)=\frac{1-f}{n} \bar{Y}\left[-\delta_{p 1} \rho C_{x} C_{y}+\delta_{p 1}^{2} C_{x}^{2}+\alpha_{1} \delta_{p 1} \rho C_{x} C_{y}-\alpha_{1} \delta_{p 1}^{2} C_{x}^{2}\right]
\end{aligned}
$$

Squaring both sides of Equation (17) and retaining the terms up to the first order of approximation, we have,

$$
\left(\xi_{p 1}-\bar{Y}\right)^{2}=\bar{Y}^{2}\left[e_{0}^{2}+\delta_{p 1}^{2} e_{1}^{2}-2 \delta_{p 1} e_{0} e_{1}+\alpha_{1}^{2} \delta_{p 1}^{2} e_{1}^{2}+2 \alpha_{1} \delta_{p 1} e_{0} e_{1}-2 \alpha_{1} \delta_{p 1}^{2} e_{1}^{2}\right]
$$

Taking expectation on both sides of Equation (19) and putting the values of different expectations, we get the mean square error of $\xi_{p 1}$, up to the first order of approximation, as

$$
\begin{aligned}
& E\left(\xi_{p 1}-\bar{Y}\right)^{2}=\operatorname{MSE}\left(\xi_{p 1}\right)=\bar{Y}^{2} E\left[e_{0}^{2}+\delta_{p 1}^{2} e_{1}^{2}-2 \delta_{p 1} e_{0} e_{1}+\alpha_{1}^{2} \delta_{p 1}^{2} e_{1}^{2}+2 \alpha_{1} \delta_{p 1} e_{0} e_{1}-2 \alpha_{1} \delta_{p 1}^{2} e_{1}^{2}\right] \\
& \left.\operatorname{MSE}\left(\xi_{p 1}\right)=\frac{1-f}{n} \bar{Y}^{2}\left[C_{y}^{2}+\delta_{p 1}^{2} C_{x}^{2}-2 \delta_{p 1} \rho C_{x} C_{y}+\alpha_{1}^{2} \delta_{p 1}^{2} C_{x}^{2}+2 \alpha_{1} \delta_{p 1} \rho C_{x} C_{y}-2 \alpha_{1} \delta_{p 1}^{2} C_{x}^{2}\right] 20\right)
\end{aligned}
$$

which is minimum for $\alpha_{1}$ when Equation (20) is partially differentiated with respect to $\alpha_{1}$ and equate to zero, we have

$$
\begin{aligned}
& \frac{\partial M S E\left(\xi_{p 1}\right)}{\alpha_{1}}=\frac{1-f}{n} \bar{Y}^{2}\left[2 \alpha_{1} \delta_{p 1}^{2} C_{x}^{2}+2 \delta_{p 1} \rho C_{x} C_{y}-2 \delta_{p 1}^{2} C_{x}^{2}\right]=0 \\
& \alpha_{1} \delta_{p 1}^{2} C_{x}^{2}=\delta_{p 1}^{2} C_{x}^{2}-\delta_{p 1} \rho C_{x} C_{y} \\
& \alpha_{p 1}=\frac{\delta_{p 1}^{2} C_{x}^{2}-\delta_{p 1} \rho C_{x} C_{y}}{\delta_{p 1}^{2} C_{x}^{2}}=\frac{A_{1}}{B_{1}}
\end{aligned}
$$

Thus, the minimum MSE of $\xi_{p 1}$ is,

$$
M S E_{\min }\left(\xi_{p 1}\right)=\frac{1-f}{n} \bar{Y}^{2}\left[C_{y}^{2}+\delta_{p 1}^{2} C_{x}^{2}-2 \delta_{p 1} \rho C_{x} C_{y}-\frac{A_{1}^{2}}{B_{1}}\right]
$$

Similarly, the biases and mean squared errors (MSE) of others proposed estimators can be obtained in the same way. Thus, the following are generalized biases and mean squared errors (MSE) of the proposed estimators given by

$$
\begin{aligned}
& B\left(\xi_{p i}\right)=\frac{1-f}{n} \bar{Y}\left[-\delta_{p i} \rho C_{x} C_{y}+\delta_{p i}^{2} C_{x}^{2}+\alpha_{i} \delta_{p i} \rho C_{x} C_{y}-\alpha_{i} \delta_{p i}^{2} C_{x}^{2}\right] \\
& M S E_{\text {min }}\left(\xi_{p i}\right)=\frac{1-f}{n} \bar{Y}^{2}\left[C_{y}^{2}+\delta_{p i}^{2} C_{x}^{2}-2 \delta_{p i} \rho C_{x} C_{y}-\frac{A_{i}^{2}}{B_{i}}\right]
\end{aligned}
$$


Where,

$$
\alpha_{i}=\frac{\delta_{p i}^{2} C_{x}^{2}-\delta_{p i} \rho C_{x} C_{y}}{\delta_{p i}^{2} C_{x}^{2}}=\frac{A_{i}}{B_{i}}, i=1,2, \ldots, 7 .
$$

Thus, biases and mean squared errors (MSE) of the proposed estimators are given as:

$$
\begin{aligned}
& B\left(\xi_{p i}\right)=\gamma \bar{Y}\left[-\delta_{p i} \rho C_{x} C_{y}+\delta_{p i}^{2} C_{x}^{2}+\alpha_{i} \delta_{p i} \rho C_{x} C_{y}-\alpha_{i} \delta_{p i}^{2} C_{x}^{2}\right] \\
& M S E_{\min }\left(\xi_{p i}\right)=\gamma \bar{Y}^{2}\left[C_{y}^{2}+\delta_{p i}^{2} C_{x}^{2}-2 \delta_{p i} \rho C_{x} C_{y}-\frac{A_{i}^{2}}{B_{i}}\right]
\end{aligned}
$$

Where, $\alpha_{i}=\frac{\delta_{p i}^{2} C_{x}^{2}-\delta_{p i} \rho C_{x} C_{y}}{\delta_{p i}^{2} C_{x}^{2}}=\frac{A_{i}}{B_{i}}, i=1,2, \ldots, 7$.

\section{Theoretical efficiency comparison}

In this section, the proposed modified ratio estimators were compared theoretically with the other existing related ratio estimators of population mean in terms of their variances and mean squared errors (MSE) under simple random sampling without replacement scheme and thereby establishing their efficiency conditions.

\section{Efficiency condition of $\xi_{p i}(i=1,2, \ldots, 7)$ over some related existing ratio estimators}

From the MSE of proposed modified ratio estimator $\xi_{p i}$ and Equation (2), proposed modified ratio estimator $\xi_{p i}$ is better than the mean per unit estimator if,

$$
\begin{gathered}
V(\bar{y})-M S E_{\min }\left(\xi_{p i}\right)=\gamma \bar{Y}^{2}\left[\delta_{p i}^{2} C_{x}^{2}-2 \delta_{p i} \rho C_{x} C_{y}-\frac{A_{i}^{2}}{B_{i}}\right]>0 \\
\text { Or, } \delta_{p i}^{2} C_{x}^{2}-2 \delta_{p i} \rho C_{x} C_{y}>\frac{A_{i}^{2}}{B_{i}} \quad(i=1,2, \ldots, 7)
\end{gathered}
$$

When Equation (24) is satisfied, $\xi_{p i}$ is more efficient than $\bar{y}$.

From the MSE of proposed modified ratio estimator $\xi_{p i}$ and Equation (2), proposed modified ratio estimator $\xi_{p i}$ is better than the usual ratio estimator $\bar{y}_{r}$ by Cochran (1940) if,

$$
\begin{aligned}
& \operatorname{MSE}\left(\hat{\bar{Y}}_{r}\right)-M S E_{\min }\left(\xi_{p i}\right)=\gamma \bar{Y}^{2}\left[\left(R^{2}-\delta_{p i}^{2}\right) C_{x}^{2}-2\left(R-\delta_{p i}\right) \rho C_{x} C_{y}-\frac{A_{i}^{2}}{B_{i}}\right]>0 \\
& \operatorname{Or},\left(R^{2}-\delta_{p i}^{2}\right) C_{x}^{2}-2\left(R-\delta_{p i}\right) \rho C_{x} C_{y}>\frac{A_{i}^{2}}{B_{i}} \quad(i=1,2, \ldots, 7)
\end{aligned}
$$

When Equation (25) is satisfied, $\xi_{p i}$ is more efficient than $\bar{y}_{r}$.

From the MSE of proposed modified ratio estimator $\xi_{p i}$ and MSE in Tables 1 and 2, proposed modified ratio estimator $\xi_{p i}$ is better than the modified existing ratio type estimator by Sisodia and Dwivedi (1981), Upadhyaya and Singh (1999), Singh and Tailor (2003), Singh et al. (2004), Yan and Tian (2010), Subramani and Kumarpandiyan (2013) and Jerajuddin and Kishun (2016) if,

$$
\operatorname{MSE}\left(\hat{\bar{Y}}_{i}\right)-M S E_{\min }\left(\xi_{p i}\right)=\gamma \bar{Y}^{2}\left[\left(R^{2}-\delta_{p i}^{2}\right) C_{x}^{2}-2\left(R-\delta_{p i}\right) \rho C_{x} C_{y}-\frac{A_{i}^{2}}{B_{i}}\right]>
$$


Or, $\left(R^{2}-\delta_{p i}^{2}\right) C_{x}^{2}-2\left(R-\delta_{p i}\right) \rho C_{x} C_{y}>\frac{A_{i}^{2}}{B_{i}}, \quad i=1,2, \ldots, 7$

Table 2: Biases and mean squared errors (MSEs) of the proposed modified ratio estimators

\begin{tabular}{|c|c|c|c|c|c|}
\hline $\mathrm{S} / \mathrm{N}$ & Estimato & Constant & Bias & MSE & \\
\hline 1. & $\xi_{p 1}$ & $\delta_{p 1}=\frac{\bar{X}}{\bar{X}+C_{x} n}$ & $\gamma \bar{Y}\left[\begin{array}{l}-\delta_{p 1} \rho C_{x} C_{y}+\delta_{p 1}^{2} C_{x}^{2} \\
+\alpha_{1} \delta_{p 1} \rho C_{x} C_{y}-\alpha_{1} \delta_{p 1}^{2} C_{x}^{2}\end{array}\right]$ & $\gamma \bar{Y}^{2}$ & $\left.\begin{array}{l}C_{y}^{2}+\delta_{p 1}^{2} C_{x}^{2} \\
-2 \delta_{p 1} \rho C_{x} C_{y}-\frac{A_{1}^{2}}{B_{1}}\end{array}\right]$ \\
\hline 2. & $\xi_{p 2}$ & $\delta_{p 2}=\frac{\bar{X} C_{x}}{\bar{X} C_{x}+\beta_{2} n}$ & $\gamma \bar{Y}\left[\begin{array}{l}-\delta_{p 2} \rho C_{x} C_{y}+\delta_{p 2}^{2} C_{x}^{2} \\
+\alpha_{2} \delta_{p 2} \rho C_{x} C_{y}-\alpha_{2} \delta_{p 2}^{2} C_{x}^{2}\end{array}\right]$ & $\gamma \bar{Y}^{2}$ & $\left.\begin{array}{l}C_{y}^{2}+\delta_{p 2}^{2} C_{x}^{2} \\
-2 \delta_{p 2} \rho C_{x} C_{y}-\frac{A_{2}^{2}}{B_{2}}\end{array}\right]$ \\
\hline 3. & $\xi_{p 3}$ & $\delta_{p 3}=\frac{\bar{X}}{\bar{X}+\rho n}$ & $\gamma \bar{Y}\left[\begin{array}{l}-\delta_{p 3} \rho C_{x} C_{y}+\delta_{p 3}^{2} C_{x}^{2} \\
+\alpha_{3} \delta_{p 3} \rho C_{x} C_{y}-\alpha_{3} \delta_{p 3}^{2} C_{x}^{2}\end{array}\right]$ & $\gamma \bar{Y}^{2}$ & $\left.\begin{array}{l}C_{y}^{2}+\delta_{p 3}^{2} C_{x}^{2} \\
-2 \delta_{p 3} \rho C_{x} C_{y}-\frac{A_{3}^{2}}{B_{3}}\end{array}\right]$ \\
\hline 4. & $\xi_{p 4}$ & $\delta_{p 4}=\frac{\bar{X}}{\bar{X}+\beta_{2} n}$ & $\gamma \bar{Y}\left[\begin{array}{l}-\delta_{p 4} \rho C_{x} C_{y}+\delta_{p 4}^{2} C_{x}^{2} \\
+\alpha_{4} \delta_{p 4} \rho C_{x} C_{y}-\alpha_{4} \delta_{p 4}^{2} C_{x}^{2}\end{array}\right.$ & $\gamma \bar{Y}^{2}$ & $\left.\begin{array}{l}C_{y}^{2}+\delta_{p 4}^{2} C_{x}^{2} \\
-2 \delta_{p 4} \rho C_{x} C_{y}-\frac{A_{4}^{2}}{B_{4}}\end{array}\right]$ \\
\hline 5. & $\xi_{p 5}$ & $\delta_{p 5}=\frac{\bar{X}}{\bar{X}+\beta_{1} n}$ & $\gamma \bar{Y}\left[\begin{array}{l}-\delta_{p 5} \rho C_{x} C_{y}+\delta_{p 5}^{2} C_{x}^{2} \\
+\alpha_{5} \delta_{p 5} \rho C_{x} C_{y}-\alpha_{5} \delta_{p 5}^{2} C_{x}^{2}\end{array}\right]$ & $\gamma \bar{Y}^{2}$ & $\left.\begin{array}{l}C_{y}^{2}+\delta_{p 5}^{2} C_{x}^{2} \\
-2 \delta_{p 5} \rho C_{x} C_{y}-\frac{A_{5}^{2}}{B_{5}}\end{array}\right]$ \\
\hline 6. & $\xi_{p 6}$ & $\delta_{p 6}=\frac{\bar{X}}{\bar{X}+M_{d} n}$ & $\gamma \bar{Y}\left[\begin{array}{l}-\delta_{p 6} \rho C_{x} C_{y}+\delta_{p 6}^{2} C_{x}^{2} \\
+\alpha_{6} \delta_{p 6} \rho C_{x} C_{y}-\alpha_{6} \delta_{p 6}^{2} C_{x}^{2}\end{array}\right]$ & $\gamma \bar{Y}^{2}$ & $\left.\begin{array}{l}C_{y}^{2}+\delta_{p 6}^{2} C_{x}^{2} \\
-2 \delta_{p 6} \rho C_{x} C_{y}-\frac{A_{6}^{2}}{B_{6}}\end{array}\right]$ \\
\hline 7 & $\xi_{p 7}$ & $\delta_{p 7}=\frac{\bar{X}}{\bar{X} M_{d}+n}$ & $\gamma \bar{Y}\left[\begin{array}{l}-\delta_{p 7} \rho C_{x} C_{y}+\delta_{p 7}^{2} C_{x}^{2} \\
+\alpha_{7} \delta_{p 7} \rho C_{x} C_{y}-\alpha_{7} \delta_{p 7}^{2} C_{x}^{2}\end{array}\right.$ & $\gamma \bar{Y}^{2}$ & $\begin{array}{l}C_{y}^{2}+\delta_{p 7}^{2} C_{x}^{2} \\
-2 \delta_{p 7} \rho C_{x} C_{y}-\frac{A_{7}^{2}}{B_{7}}\end{array}$ \\
\hline
\end{tabular}

\section{Dataset for empirical study}

To judge the performance of the proposed modified ratio estimators and the existing related ratio estimators of population mean using auxiliary variable, we considered four natural populations from two sources. First two populations, populations 1 and 2 are from Murthy (1967), while populations 3 and 4 are from Mukhopadhyay (2009).

\section{Murthy (1967)}

Population 1: $Y=$ Output for 80 factories in a region and $X=$ Number of workers

$N=80, n=20, \bar{Y}=51.8264, \bar{X}=11.2646$, $\rho=0.9413, C_{y}=0.3542, C_{x}=0.7507$ $\beta_{1}=1.0500, \beta_{2}=-0.0634, M_{d}=7.5750$.

Population 2: $Y=$ Output for 80 factories in a region and $X=$ Fixed Capital

$N=80, n=20, \bar{Y}=51.8264$,

$\bar{X}=11.2646, \rho=0.9413$,

$C_{y}=0.3542, C_{x}=0.9485$

$\beta_{1}=1.3006, \beta_{2}=0.6977, M_{d}=1.4800$

\section{Mukhopadhyay (2009)}

Population 3: $Y=$ Output for 40 factories in a region and $X=$ Number of workers

$N=40, n=8, \bar{Y}=50.7858, \bar{X}=2.3033$,

$\rho=0.8006, C_{y}=0.3295, C_{x}=0.8406$ 
$\beta_{1}=0.8799, \beta_{2}=-0.4622, M_{d}=1.2500$.

Population 4: $Y=$ Output for 40 factories in a region and $X=$ Fixed capital

$N=40, n=8, \bar{Y}=50.7858, \bar{X}=9.4543$,

$\rho=0.8349, C_{y}=0.3295, C_{x}=0.6756$

$\beta_{1}=0.8799, \beta_{2}=-0.4622, M_{d}=7.0700$.

\section{Results and Discussion}

In this section, the performances of the proposed ratio estimators are evaluated and compared with the mentioned ratio estimators in Table 1 by using the population data of Murthy (1967) and Mukhopadhyay (2009). We apply the proposed and existing estimators to this data set, and the efficiency of the proposed modified ratio estimators over some existing related ratio estimators were investigated using real life data to support the theoretical comparisons in the previous section of this paper.

The numerical values of biases and the mean squared errors as well as percentage relative efficiency (PRE) of the newly proposed modified ratio estimators over other existing related ratio estimators of population mean using auxiliary variable for the four populations are as shown in Tables 3-6.

From Tables 3-6, it can be observed that some proposed modified ratio estimators are having lower biases when compared with other existing related ratio estimators, while the mean squared errors of the newly proposed modified ratio estimators were also lower as compared to other existing related ratio estimators.

Table 3: Biases, mean squared errors and percentage relative efficiency of the existing and newly proposed modified ratio estimators using population 1

\begin{tabular}{|c|c|c|c|c|}
\hline Estimator & Constant & Bias & MSE & PRE \\
\hline $\bar{y}$ & 0.000000 & 0.000000 & 12.63661 & NA \\
\hline$\hat{\bar{Y}}_{r}$ & 0.000000 & 0.608819 & 18.97931 & 66.581 \\
\hline$\hat{\bar{Y}}_{1}$ & 0.937521 & 0.050583 & 15.25812 & 82.819 \\
\hline$\hat{\bar{Y}}_{2}$ & 1.007554 & 0.131644 & 19.45925 & 64.939 \\
\hline$\hat{\bar{Y}}_{3}$ & 0.922882 & 0.034995 & 14.450269 & 87.448 \\
\hline$\hat{\bar{Y}}_{4}$ & 1.005660 & 0.129311 & 19.33831 & 65.345 \\
\hline$\hat{\bar{Y}}_{5}$ & 0.914735 & 0.026525 & 14.01128 & 90.189 \\
\hline$\hat{\bar{Y}}_{6}$ & 0.597921 & -0.190136 & 2.782544 & 454.139 \\
\hline$\hat{\bar{Y}}_{7}$ & 0.360299 & -0.208344 & 1.838908 & 687.180 \\
\hline$\xi_{p 1}$ & 0.428661 & -0.007525 & 1.439881 & 877.615 \\
\hline$\xi_{p 2}$ & 1.176397 & 0.3562010 & 1.439996 & 877.545 \\
\hline$\xi_{p 3}$ & 0.374356 & -0.033941 & 1.439885 & 877.612 \\
\hline$\xi_{p 4}$ & 1.126843 & 0.332096 & 1.439992 & 877.547 \\
\hline$\xi_{p 5}$ & 0.349132 & -0.046211 & 1.439985 & 877.552 \\
\hline$\xi_{p 6}$ & 0.069208 & -0.182376 & 1.439981 & 877.554 \\
\hline$\xi_{p 7}$ & 0.810119 & 0.1780302 & 1.439988 & 877.550 \\
\hline
\end{tabular}


Table 4: Biases, mean squared errors and percentage relative efficiency of the existing and newly proposed modified ratio estimators using population 2

\begin{tabular}{lllll}
\hline Estimator & Constant & Bias & MSE & PRE \\
\hline $\bar{y}$ & 0.000000 & 0.000000 & 12.63661 & NA \\
$\hat{\bar{Y}}_{r}$ & 0.000000 & 1.151032 & 41.32765 & 30.577 \\
$\hat{\bar{Y}}_{1}$ & 0.937521 & 0.087907 & 17.19251 & 73.501 \\
$\hat{\bar{Y}}_{2}$ & 1.007554 & 0.155035 & 20.671513 & 61.131 \\
$\hat{\bar{Y}}_{3}$ & 0.922882 & 0.097524 & 17.690914 & 71.430 \\
$\hat{\bar{Y}}_{4}$ & 1.005660 & 0.168609 & 21.375015 & 59.119 \\
$\hat{\bar{Y}}_{5}$ & 0.914735 & 0.004041 & 12.84606 & 98.370 \\
$\hat{\bar{Y}}_{6}$ & 0.597921 & -0.028866 & 11.140575 & 113.429 \\
$\hat{\bar{Y}}_{7}$ & 0.360299 & -0.121869 & 6.320581 & 199.928 \\
$\xi_{p 1}$ & 0.130666 & -0.126073 & 2.056924 & 614.345 \\
$\xi_{p 2}$ & 0.162347 & -0.107145 & 2.056889 & 614.355 \\
$\xi_{p 3}$ & 0.134805 & -0.123600 & 2.056895 & 614.354 \\
$\xi_{p 4}$ & 0.169667 & -0.1027724 & 2.056881 & 614.358 \\
$\xi_{p 5}$ & 0.098786 & -0.1451187 & 2.056933 & 614.342 \\
$\xi_{p 6}$ & 0.087864 & -0.1516441 & 2.056947 & 614.338 \\
$\xi_{p 7}$ & 0.174234 & -0.100044 & 2.056875 & 614.359 \\
\hline & & & &
\end{tabular}


Suleiman and Adewara - Improved Modified Ratio Estimation of Population Mean ...

Table 5: Biases, mean squared errors and percentage relative efficiency of the existing and newly proposed modified ratio estimators using population 3

\begin{tabular}{lllll}
\hline Estimator & Constant & Bias & MSE & PRE \\
\hline $\bar{y}$ & 0.000000 & 0.000000 & 28.0024 & NA \\
$\hat{\bar{Y}}_{r}$ & 0.000000 & 2.46240 & 95.86411 & 29.211 \\
$\hat{\bar{Y}}_{1}$ & 0.937521 & 0.276010 & 42.01979 & 66.641 \\
$\hat{\bar{Y}}_{2}$ & 1.007554 & 3.735316 & 217.7034 & 12.863 \\
$\hat{\bar{Y}}_{3}$ & 0.922882 & 0.304709 & 43.47728 & 64.407 \\
$\hat{\bar{Y}}_{4}$ & 1.005660 & 3.151586 & 188.05822 & 14.890 \\
$\hat{\bar{Y}}_{5}$ & 0.914735 & 0.189565 & 37.62961 & 74.416 \\
$\hat{\bar{Y}}_{6}$ & 0.597921 & 0.0478559 & 30.43281 & 92.014 \\
$\hat{\bar{Y}}_{7}$ & 0.360299 & -0.324172 & 11.53909 & 242.674 \\
$\xi_{p 1}$ & 0.255126 & -0.0661004 & 10.053897 & 278.523 \\
$\xi_{p 2}$ & -0.827754 & -1.285603 & 10.053953 & 278.521 \\
$\xi_{p 3}$ & 0.264501 & -0.0555424 & 10.053889 & 278.523 \\
$\xi_{p 4}$ & -1.168061 & -1.668846 & 10.053966 & 278.521 \\
$\xi_{p 5}$ & 0.2281557 & -0.096473 & 10.053941 & 278.522 \\
$\xi_{p 6}$ & 0.1872099 & -0.142585 & 10.053976 & 278.521 \\
$\xi_{p 7}$ & 0.2646467 & -0.0553781 & 10.053876 & 278.523 \\
\hline & & & & \\
\hline & & & & \\
\hline
\end{tabular}


Table 6: Biases, mean squared errors and percentage relative efficiency of the existing and newly proposed modified ratio estimators using population 4

\begin{tabular}{lllll}
\hline Estimator & Constant & Bias & MSE & PRE \\
\hline $\bar{y}$ & 0.000000 & 0.00000 & 28.0024 & NA \\
$\hat{\bar{Y}}_{r}$ & 0.000000 & 1.37415 & 49.85359 & 56.169 \\
$\hat{\bar{Y}}_{1}$ & 0.937521 & 0.257278 & 41.06847 & 68.185 \\
$\hat{\bar{Y}}_{2}$ & 1.007554 & 0.658753 & 61.45774 & 45.564 \\
$\hat{\bar{Y}}_{3}$ & 0.922882 & 0.222518 & 69.30314 & 40.406 \\
$\hat{\bar{Y}}_{4}$ & 1.005660 & 0.577651 & 57.33886 & 48.837 \\
$\hat{\bar{Y}}_{5}$ & 0.914735 & 0.213064 & 38.82301 & 72.128 \\
$\hat{\bar{Y}}_{6}$ & 0.597921 & -0.321274 & 11.68628 & 239.618 \\
$\hat{\bar{Y}}_{7}$ & 0.360299 & -0.342432 & 10.61172 & 263.882 \\
$\xi_{p 1}$ & 0.636263 & 0.216217 & 8.483064 & 330.098 \\
$\xi_{p 2}$ & 2.374713 & 1.857124 & 8.483106 & 330.096 \\
$\xi_{p 3}$ & 0.586004 & 0.168778 & 8.483052 & 330.098 \\
$\xi_{p 4}$ & 1.642312 & 1.165818 & 8.483099 & 330.096 \\
$\xi_{p 5}$ & 0.573214 & 0.1567055 & 8.483031 & 330.099 \\
$\xi_{p 6}$ & 0.143216 & -0.249166 & 8.483006 & 330.100 \\
$\xi_{p 7}$ & 0.893108 & 0.4586518 & 8.483088 & 330.097 \\
\hline & & & &
\end{tabular}

\section{Conclusion}

From the results of empirical study using four natural population datasets, it can be concluded that the newly proposed modified ratio estimators in this study demonstrated high relative efficiency over existing related ratio estimators. From Table 3, all the newly proposed modified ratio estimators has PRE of about 877.6 which is higher than the PRE of all the existing related ratio estimations. This is also the case in Tables 4-6, where all the newly proposed modified ratio estimators have PRE of about 614.3 (Table 4), 278.5 (Table 5) and 330.1 (Table 6), respectively, which are higher than the PRE of all the existing related ratio estimations. In population 1 , the newly proposed modified ratio estimator $\xi_{p 1}$ is the most efficient estimator with PRE of 877.615 , followed by $\xi_{p 3}, \xi_{p 6}, \xi_{p 5}, \xi_{p 7}, \xi_{p 4}$, and $\xi_{p 2}$ in that order. Also, in population 2, the newly proposed modified ratio estimator $\xi_{p 7}$ is the most efficient estimator with PRE of 614.359, followed by $\xi_{p 4}, \xi_{p 2}, \xi_{p 3}, \xi_{p 1}, \xi_{p 5}$ and $\xi_{p 6}$ in that order. Moreover, in population 3, the newly proposed modified ratio estimators $\xi_{p 1}, \quad \xi_{p 3}$ and $\xi_{p 7}$ are the most efficient estimators with PRE of 278.523, followed by $\xi_{p 5}$, then, $\xi_{p 2}, \xi_{p 4}$, and $\xi_{p 6}$. Finally, in 
population 4 , the newly proposed modified ratio estimator $\xi_{p 6}$ is the most efficient estimator with PRE of 330.100 , followed by $\xi_{p 5}$, then $\xi_{p 1}$ and $\xi_{p 3}, \xi_{p 7}, \xi_{p 2}$ and $\xi_{p 4}$, and in that order. In conclusion, the newly proposed modified ratio estimators are improved versions of Gupta and Yadav (2018) generalized estimator of population mean using information on size of the sample. Based on the empirical findings, the newly proposed modified ratio estimators are recommended for estimating finite population mean of any variable of interest.

\section{References}

Abid M, Abbas N, Sherwani RAK and Nazir HZ 2016 Improved ratio estimators for the population mean using non-conventional measure of dispersion. Pak. J. Stat. Oper. Res. XII (2): 353-367.

Cochran WG 1940 The estimation of the yields of the cereal experiments by sampling for the ratio of grain to total produce. J. Agric. Sci. 30: 262-275.

Cochran WG 1977 Sampling Techniques. John Wiley and Sons, New York.

Gupta RK and Misra S 2006 Estimation of population variance using ratio type estimator in Indian J. Math. Mathemat. Sci. 2(2): 169-176.

Gupta RK and Yadav SK 2017 New efficient estimators of population mean using nontraditional measures of dispersion. Open J. Stat. 7(3): 394-404.

Gupta RK and Yadav SK 2018 Improved estimation of population mean using information on size of the sample. Am. J. Math. Stat. 8(2): 27-35.

Jerajuddin M and Kishun J 2016 Modified ratio estimators for population mean using size of the sample selected from population. IJSRSET 2(2): 10-16.

Kadilar C and Cingi H 2004 Ratio estimators in simple random sampling. Appl. Math. Comput. 151: 893-902.

Kadilar C and Cingi H 2006a An improvement in estimating the population mean by using the correlation co-efficient. Hacettepe J. Math. Stat. 35(1): 103-109.
Kadilar C and Cingi, H 2006b Improvement in estimating the population mean in simple random sampling. Appl. Math. Lett. 19: 75-79.

Kadilar C and Cingi H 2009 Advances in sampling theory-ratio method of estimation, Bentham Science Publishers.

Koyuncu N and Kadilar C 2009 Efficient estimators for the population mean. Hacettepe J. Math. Stat. 38(2): 217-225.

Misra S and Gupta RK 2008 Almost unbiased jacknifed ratio type estimator of population variance. Int. J. Agric. Stat. Sci. 4: 345-350.

Misra S, Gupta RK and Shukla AK 2012 Generalized class of estimators for estimation of finite population variance. Int. J. Agric. Stat. Sci. 8(2): 447-458.

Mukhopadhyay P 2009 Theory and methods of survey sampling. PHI Learning, $2^{\text {nd }}$ edition, New Delhi.

Murthy MN 1967 Sampling Theory and Methods. Statistical Publishing Society, Calcutta.

Singh GN 2003 On the improvement of product method of estimation in sample surveys. J. Indian Soc. Agric. Stat. 56(3): 267-265.

Singh HP, Upadhyaya, LN and Chandra P 2004 A general family of estimators for estimating population mean using two auxiliary variables in two-phase sampling. Stat. Transit. 6(7): 1055-1077.

Singh D and Chaudhary FS 1986 Theory and analysis of sample survey designs. New Age International Publisher, New Delhi.

Singh HP and Tailor R 2003 Use of known correlation coefficient in estimating the finite population means. Stat. Transit. 6(4): 555-560.

Singh HP and Tailor R 2005 Estimation of finite population mean with known coefficient of variation of an auxiliary variable. Statistica. anno LXV. 3: 301313.

Sisodia BVS and Dwivedi VK 1981 A Modified ratio estimator using coefficient of variation of auxiliary variable. $J$. Indian. Soc. Agri. Stat. 33(1): 13-18.

Subramani J 2013a Generalized modified ratio estimator of finite population mean. 
J. Modern Appl. Stat. Meth. 12(2): 121155.

Subramani J and Kumarapandiyan G 2012a Estimation of population mean using coefficient of variation and median of an auxiliary variable. Int. J. Prob. Stat. 1(4): 111-118.

Subramani J, and Kumarapandiyan G 2012b Modified ratio estimators using known median and coefficient of kurtosis. Am. J. Math. Stat. 2(4): 95-100.

Subramani J and Kumarapandiyan G 2012c Estimation of population mean using known median and coefficient of skewness. Am. J. Math. Stat. 2(5): 101107.

Subramani J and Kumarapandiyan G 2013a Estimation of population mean using deciles of an auxiliary variable. Statistics in Transition-New Series 14(1): 75-88.

Subramani J 2013b A new modified ratio estimator of population mean when median of the auxiliary variable is known. Pak. J. Stat. Oper. Res. 9(2): 137-145.

Tailor R, Parmar R, Kim JM and Tailor R 2011 Ratio-cum-product estimators of population mean using known population parameters of auxiliary variates. Commun. Stat. Appl. Methods 18(2): 155-164.

Tailor R and Sharma B 2009 A modified ratio-cum-product estimator of finite population mean using known coefficient of variation and coefficient of kurtosis. Stat. Transit.-New Ser. 10(1): 15-24.

Upadhyaya LN and Singh HP 1999 Use of transformed auxiliary variable in estimating the finite population means. Biom. J. 41(5): 627-636.

Yadav SK and Pandey H 2011 Improved exponential estimators of population mean using qualitative auxiliary information under two phase sampling. Investig. Math. Sci. 1: 85-94.
Yadav SK and Adewara AA 2013 On improved estimation of population mean using qualitative auxiliary information. Math. Theor. Model. 3(11): 42-50.

Yadav SK, Mishra SS and Shukla AK 2014 Improved ratio estimators for population mean based on median using linear combination of population mean and median of an auxiliary variable. Am. J. Oper. Res. 4(2): 21-27.

Yadav SK, Mishra, SS and Shukla, AK 2015 Estimation approach to ratio of two inventory population means in stratified random sampling. Am. J. Oper. Res. 5(4): 96-101.

Yadav SK, Mishra SS, and Shukla AK, Kumar S and Singh RS 2016a Use of nonconventional measures of dispersion for improved estimation of population mean. Am. J. Oper. Res. 6(3): 69-75.

Yadav SK, Gupta SAT, Mishra SS and Shukla AK 2016b Modified ratio and product estimators for estimating population mean in two-phase sampling. Am. J. Oper. Res. 6(3): 61-68.

Yadav SK, Subramani J, Mishra SS and Shukla AK 2016c Improved ratio-cumproduct estimators of population mean using known population parameters of auxiliary variables. Am. J. Oper. Res. 6(2): 48-54.

Yadav SK, Misra S, Mishra SS, and Chutiman N 2016d Improved ratio estimators of population mean in adaptive cluster sampling. J. Stat. Appl. Prob. Lett. 3: 1-6.

Yan Z and Tian B 2010 Ratio method to the mean estimation using coefficient of skewness of auxiliary variable. Int. Conf. Inf. Comput. Appl. 103-110. 